\title{
SCAO Simulation Results with a Pyramid Sensor on an ELT-like Telescope
}

\author{
Aurea Garcia-Rissmann ${ }^{1, a}$ and Miska Le Louarn² \\ 1 Gemini Observatory Southern Operations Center, c/o AURA, Casilla 603 La Serena, Chile \\ 2 European Southern Observatory, Karl-Schwarzschild-Strasse 2, 85748, Garching bei Muenchen, \\ Germany
}

\begin{abstract}
We show the performance derived from simulations of a single-conjugated adaptive optics (SCAO) system for an ELT using the pyramid wavefront sensor (hereafter PWFS). The main objective of this work is to obtain the parameters which optimize the Strehl and characterize the system. Adopting K as the science band, we verify that the overall performance tends to be poorer as the sensing wavelength becomes shorter (as we probed $\mathrm{K}, \mathrm{J}$ and $\mathrm{R}$ bands), the loop gain optimal range is dependent on the singular-value decomposition (SVD) truncation threshold used to build the command matrix, and that a "natural" modulation for the PWFS (i.e. zero modulation) produces in general poorer results when compared to modulated cases, being this especially true for the R-sensing band. Also more modulation is needed as one goes to shorter wavelengths. Comparisons between a scaled-down version of the simulations and those for a $42 \mathrm{~m}$ telescope are performed. The atmospheric model adopted was always a von Karman with $\mathrm{r}_{0}=0.13 \mathrm{~m} @ 500 \mathrm{~nm}$ and outer-scale $\mathrm{L}_{0}=25 \mathrm{~m}$. The telescope pupil had a central obstruction of $28 \%$ and no spiders. The NGS was put on-axis, and diffraction between pyramid imaged pupils taken into account.
\end{abstract}

\section{Introduction}

The design of adaptive optics systems for the next generation of extremely large telescopes pose several technical challenges, and therefore studying and comparing the performance of different wavefront sensors are a necessary task.

The working principle of the PWFS relies on the Foucault knife-edge test, in which a linear edge along one direction is introduced gradually in the image plane of the beam, allowing one to evaluate deviations from a perfectly unaberrated wavefront. In astronomical adaptive optics, the concept of this sensor was introduced by [5], and has been gaining importance in the state-of-the-art instrumentation because of some of its advantages in terms of sensitivity over the Shack-Hartmann WFS (SHWFS), especially in high-contrast $\mathrm{AO}$.

The formalism for the knife-edge test is explained in detail in [4]. In the context of the PWFS, the four edges of the pyramid with a square basis divides the beam and re-images the telescope pupil in four different directions (see figure 1). Computationally, there are basically two approaches for building the imaged pupils: the amplitude-mask and the phase-mask. The former builds each imaged pupil independently of the others, and could be used when their separation on the detector (dependent on the apex of the pyramid) is large. However, the phase-mask approach is more accurate in the sense that it calculates the four imaged pupils simultaneously, taking into account diffraction effects between them. The basic equation for the pyramid transmittance function under this formalism is (e.g. [2]):

$$
\mathrm{T}_{p y r}(\mathbf{f})=\sum_{n=0}^{1} \sum_{m=0}^{1} A_{p y r} H\left((-1)^{n} f_{x},(-1)^{m} f_{y}\right) \exp \left\{-i \alpha\left[(-1)^{n} f_{x}+(-1)^{m} f_{y}\right]\right\}
$$

being $H$ the 2-D Heaviside function $(H(x, y)=1$ when $x$ and $y$ are positive, and zero otherwise). $A_{p y r}$ is a complex parameter that depends on the pyramid geometry and $\alpha$ the tangent of the pyramid

\footnotetext{
a e-mail: augarcia@gemini.edu
} 

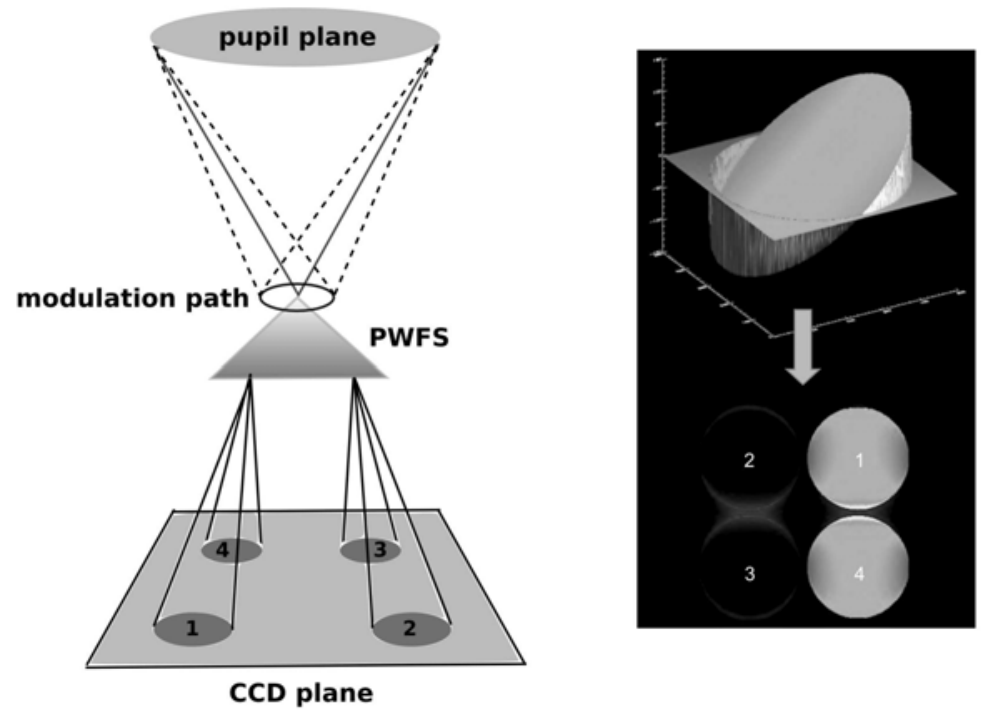

Fig. 1. Working principle of the PWFS under modulation: four imaged pupils are formed in the CCD plane, being this latter conjugated to the pupil plane of the telescope (left). On the right, an example of a "tipped" wavefront reaching the tip of the pyramid; the slopes can be estimated from the signal calculated according to equations (4) and (5).

divergence angle (as defined in [1], [6]). Given the complex amplitude of the wavefront in the pupil plane:

$$
\Psi(\mathbf{r})=P(\mathbf{r}) \exp [i \phi(\mathbf{r})]
$$

with $P$ being the pupil function, we can calculate the intensity on the detector plane as:

$$
\mathrm{I}\left(\mathbf{r}^{\prime}\right)=\left|F T^{-1}\left\{\mathrm{~T}_{p y r}(\mathbf{f})\right\} * \Psi(\mathbf{r})\right|^{2}
$$

Just like in a simple knife-edge test, a static setup for the PWFS is capable of providing only information on the sign of the wavefront reaching the tip of the pyramid. When a modulation is introduced (i.e. the signal is made to cross the edges of the sensor at a certain radius $\beta$ from the pyramid apex), the PWFS gives a linear response for spatial frequencies lower than $\beta / \lambda$. In this mode, the PWFS works as a slope sensor, behaving very similarly to a SHWFS. This occurs, as shown by [7], with a gain in sensitivity with respect to this latter of $\lambda /(2 d \beta)$, where $d$ is the sub-aperture size. But notice that in the limit of no modulation or for $|\mathbf{f}|>\beta / \lambda$, the sensor can only give the right sign of the wavefront, behaving again like a phase sensor.

Figure 1 also shows an example of the response of the PWFS for a "tipped" wavefront. Designing the system in such a way that each pixel has the projected size of a sub-aperture, the slope signal of the wavefront can then be calculated in that sub-aperture taking the correspondent pixel intensity from each imaged pupil:

$$
\begin{aligned}
& S_{x}=\left[\left(I_{1}+I_{4}\right)-\left(I_{2}+I_{3}\right)\right] / \sum I_{i} \\
& S_{y}=\left[\left(I_{1}+I_{2}\right)-\left(I_{3}+I_{4}\right)\right] / \sum I_{i}
\end{aligned}
$$

In what follows, we show the results derived from the simulations performed at ESO for different modulated cases, including a "natural" modulation $(\beta=0)$, and dependencies with other important parameters, in the context of a SCAO system. 
Table 1. Main parameters for the $42 \mathrm{~m}$ telescope simulations.

\begin{tabular}{ll}
\hline Main Parameters \\
\hline$\diamond 42 \mathrm{~m}$ telescope \\
$\diamond 28 \%$ of central obscuration, no spiders \\
$\diamond$ on-axis sensing/science target (SCAO mode) \\
$\diamond$ science band: K (5 mag star) \\
$\diamond$ square actuators geometry: 5402 \\
$\diamond 5040$ (out of 7056) active sub-apertures \\
$\diamond \quad 8 \times \lambda / D$ modulation steps \\
$\diamond$ PWFS FoV=2.5 arcsec \\
$\diamond$ von Karman atmospheric model, with $r_{0}=0.13$ m @ 500nm and $L_{0}=25 \mathrm{~m}$ \\
$\diamond 10$ simulated atmospheric layers with heights ranging from 0 to $18.5 \mathrm{~km}$ \\
$\diamond 1000$ iterations per simulation \\
\hline Variable Parameters \\
$\diamond$ loop gain $[0.2,0.4,0.6,0.8,1.0,1.2,1.4]$ \\
$\diamond$ modulation $(\beta=\lambda / D$ ) $[0,2,4,8]$ \\
$\diamond$ sensing band $[\mathrm{K}, \mathrm{J}, \mathrm{R}]$ \\
$\diamond \quad$ SVD truncation mode $[2000,2500,3000,3500,4000,4500,5000]$ \\
\hline
\end{tabular}

\section{Simulations}

Simulations for a SCAO case have been carried out using the parallelized code on the ESO beowulf cluster [3], using 70-80 machines. This program now called OCTOPUS is run by setting simulation parameters through a file that contains detailed information about the telescope and its AO system, atmospheric models, NGS asterism geometry, and other relevant input data. The specific part of the code that performs the phase-mask approach for these simulations was written by C. Vérinaud in 2006, and has as input parameters, for instance, the FoV seen by the pyramid, the separation between pupils, among others. The PWFS parameter we investigate in this work is the modulation $\beta$, given in units of $\lambda / D$ (where $\mathrm{D}$ is the telescope diameter). As a rule of thumb, the number of modulation points is set as $8 \times \beta$, so larger modulation radii require more computational time, in particular for larger systems, but at least this ensures a better accuracy in the results. The main idea of these simulations is to optimize some setup and loop parameters for this simplest case of AO, but taking into account that the telescope pupil has a central obstruction of $28 \%$.

Before starting the simulations for a $42 \mathrm{~m}$ aperture, we scaled $4 \times$ down the telescope aperture in order to have an fast estimate of the parameter regimes we should work on. These preliminary simulations were run for both a square and a radial DM actuators configuration. For the square configuration, which is analyzed in the context of this work, the corresponding number of active actuators and subapertures in the scaled-down simulations were, respectively, 386 and 304 (out of 441). The best SVD truncation mode, one of the parameters optimized here, was found to be around 320 (in the KL basis). It can be shown that there is a good qualitative agreement of these scaled-down simulations with the large simulations results. This refers to the optimal loop gains and long-exposure (LE) K-band Strehls found for each sensing band, but obviously we need to scale up the best SVD truncation mode given the increased number of actuators in the $42 \mathrm{~m}$ aperture case.

\subsection{Results from simulations for a $42 \mathrm{~m}$ aperture telescope}

The "large" simulations have the main setup parameters shown in table 1 . The number of iterations per simulation was chosen after checking the behaviour of the LE Strehl along iterations. After 1000 iterations, the LE Strehl is already stable for all the cases. 

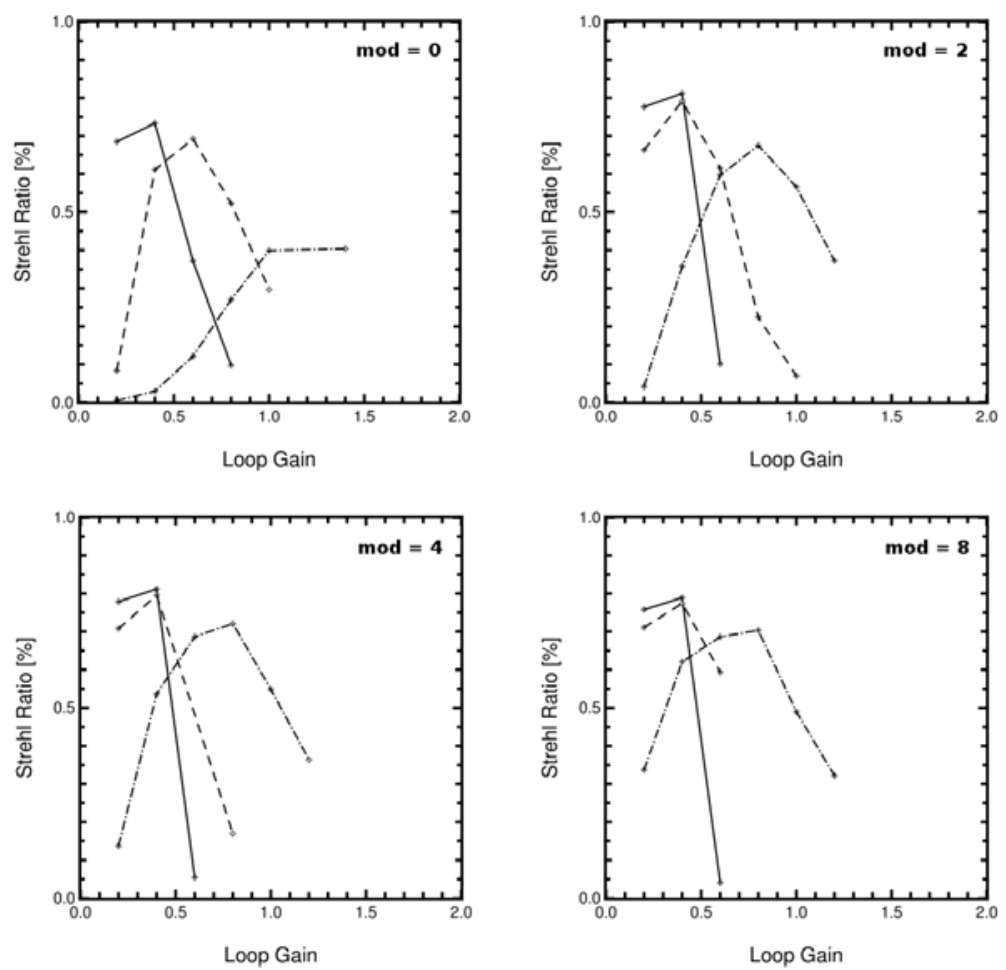

Fig. 2. LE Strehl measured in the K-band versus loop gain for 4 different modulations and a SVD truncation mode of 3000. Seeing of about $0.88 \mathrm{arcsec}$ FWHM $\left(\mathrm{r}_{0}=0.13 \mathrm{~m} @ 500 \mathrm{~nm}\right)$. Solid, dashed and dot-dashed lines refer to K, $\mathrm{J}$ and $\mathrm{R}$ sensing bands, respectively.

Table 2. Best LE Strehl results in K-band obtained from the large simulations using the parameters of table 1. LG: loop gain; SVD trunc: truncation mode.

\begin{tabular}{cccccccccc}
\hline & \multicolumn{3}{c}{ K } & \multicolumn{3}{c}{$\mathbf{J}$} & \multicolumn{3}{c}{$\mathbf{R}$} \\
\hline$\beta$ & LG & SVD trunc & Strehl (\%) & LG & SVD trunc & Strehl (\%) & LG & SVD trunc & Strehl (\%) \\
\hline 0 & 0.4 & 2500 & 75 & 0.6 & 3000 & 69 & 1.0 & 4000 & 43 \\
2 & 0.4 & 3000 & 81 & 0.4 & 4500 & 83 & 0.6 & 4000 & 68 \\
4 & 0.4 & 4000 & 84 & 0.4 & 4000 & 83 & 0.6 & 4000 & 75 \\
8 & 0.4 & 4000 & 83 & 0.4 & 4000 & 82 & 0.6 & 4000 & 76 \\
\hline
\end{tabular}

We have initially scanned through SVD truncation modes ranging from about 2000 to 5000 (not in a complete grid, but with a fairly good coverage). Having checked that the optimum range of SVD truncation modes for the final LE Strehl falls between 3000 and 4000, we decided to run the simulations mainly in this interval. The results for these two SVD truncation modes are shown in figures 2 and 3. In total, 176 simulations in the range of parameters shown in table 1 have been carried out, and the overall best results in LE K-band Strehl are shown in table 2. 

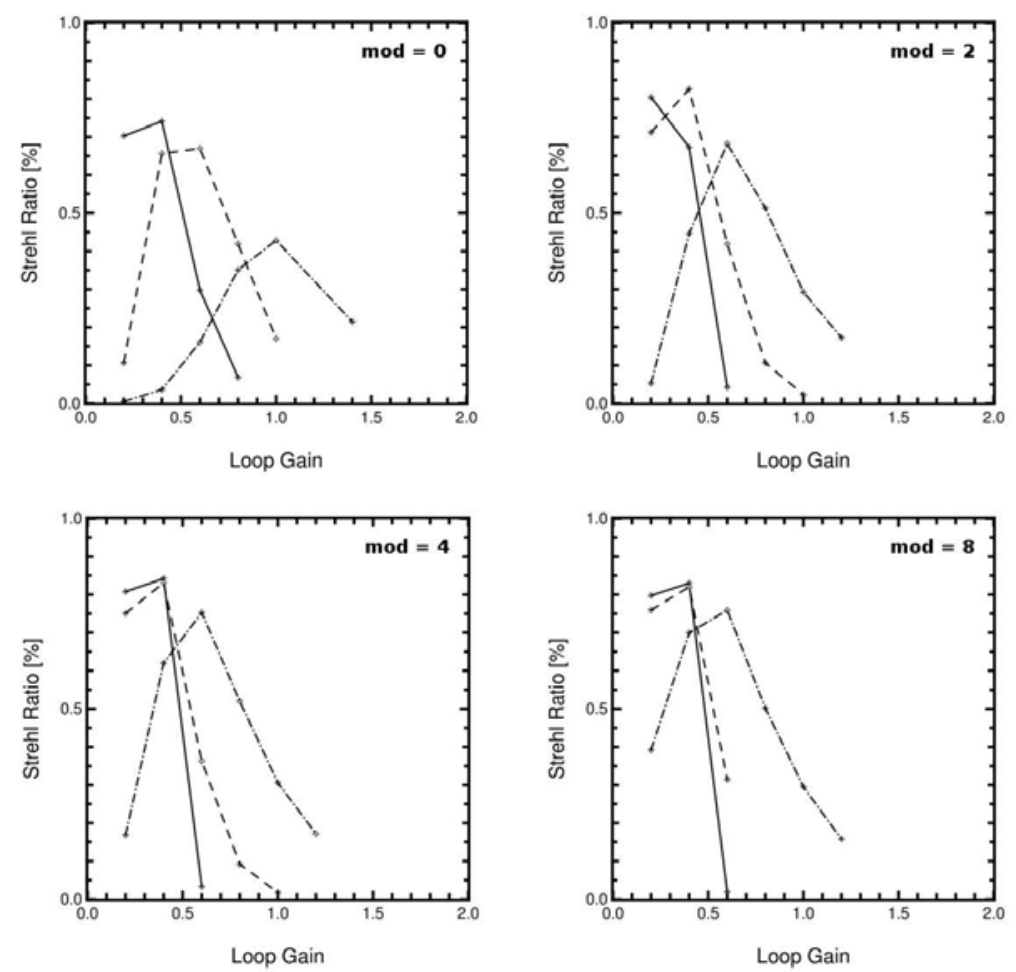

Fig. 3. Same as previous figure, but for a SVD truncation mode of 4000.

\subsection{Simulations under better and worse seeing conditions}

Simulations under different seeing conditions are ongoing, but preliminary results can be seen in figure 4 for two different modulations. This figure gives the best results in terms of LE Strehls assuming a SVD truncation mode of 4000, and shows basically a better performance in the results for the modulated case. Also, the dependence on the sensing band is less significant here. The non-modulated case requires also higher loop gains as one goes to shorter sensing wavelengths (around 1.0-1.2 for $\mathrm{R}$ band and 0.2-0.4 for $\mathrm{K}$ band).

\section{General conclusions and pespectives}

- Scaled-down simulations (using a telescope of $10.5 \mathrm{~m}$ ) give a fast estimate in terms of the optimal Strehls and loop gains necessary to achieve them with the large simulations.

- The highest LE K-band Strehls are obtained using the K-band wavefront sensing, and the performance decreases as one adopts bluer sensing bands.

- A plateau in performance is achieved in all probed sensing bands for modulations larger than about $4 \lambda / D$, and such asymptotic behaviour is reached faster the redder the used sensing bands are.

- The non-modulated case seems to show a poorer performance when compared to the other modulated cases. Also, the system takes longer to stabilize its LE K-band Strehl for zero modulation.

- The optimal loop gain varies with sensing band and slightly with modulation.

- The spread of loop gains varies with the SVD truncation mode. When comparing 3000 and 4000 SVD truncation modes, for instance, we see that the overall best performance is achieved with the latter, but the range of acceptable loop gains becomes narrower. 

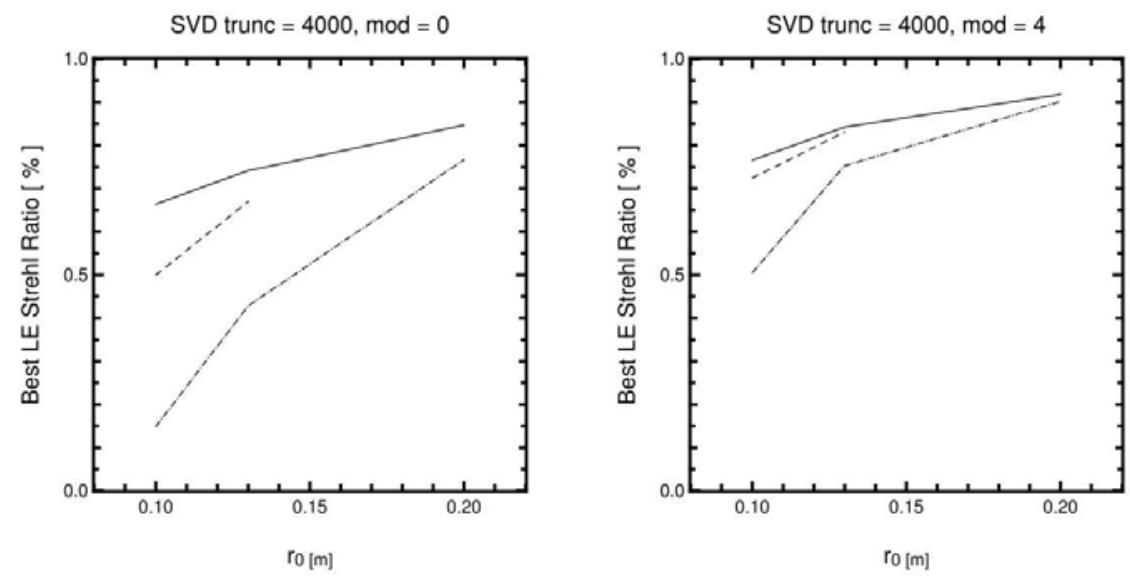

Fig. 4. Ongoing simulations: best LE K-band Strehl results versus $r_{0}$, ranging from seeings of about 0.5 to 1.0 arcsec FWHM (@500nm). Solid, dashed and dot-dashed lines correspond to K, J and R sensing bands, respectively.

- Simulations under different seeing conditions show that for a fixed SVD truncation mode (4000) a better stability and performance is achieved under a modulated case. Non-modulated cases require a larger range of loop gains to achieve better performances under different sensing bands.

A natural extension of this work would be to continue to probe the performance of the PWFS in other atmospheric conditions (better and poorer) and modulations. It would be interesting to conduct a study with a radial geometry for the actuators as well.

AGR acknowledges the support of the European Communities Commission Seventh Framework Programme E-ELT Prep (Grant INFRA-2007-2.2.1.28), in the form of a travel grant which enabled her to attend this conference.

\section{References}

1. Arcidiacono C., Optics Communications 252, (2005) 239-246.

2. Korkiakoski V., PhD Thesis, University of Helsinki, (2008), Appendix A.

3. Le Louarn M., Vérinaud C., Korkiakoski V., Fedrigo E. Advancements in Adaptive Optics (Bonaccini Calia D., Ellerbroek B.L., Ragazzoni R., 2004) Volume 5490 of SPIE Proc., 705-712.

4. Linfoot E.H., Proceedings of the Royal Society of London. Series A, Mathematical and Physical Sciences 193, (1948) 248-259.

5. Ragazzonni R., Journal of Modern Optics 43, (1996) 289-293.

6. Riccardi A., Bindi N., Ragazzoni R. Esposito S., Stefanini P. Adaptive Optical System Technologies

(Bonaccini Calia D., Tyson R.K., 1998) Volume 3353 of SPIE Proc., 941-951.

7. Vérinaud C., Optics Communications 233, (2004) 27-38. 